\title{
Nuclear Magnetic Resonance Study of Reorientational Motion in $\alpha-\operatorname{Mg}\left(\mathrm{BH}_{4}\right)_{2}$
}

\author{
Alexander V. Skripov, ${ }^{*}{ }^{\dagger}$ Alexei V. Soloninin, ${ }^{\dagger}$ Olga A. Babanova, ${ }^{\dagger}$ Hans Hagemann,,${ }^{\ddagger}$ and \\ Yaroslav Filinchuk ${ }^{\S}$ \\ Institute of Metal Physics, Ural Division of the Russian Academy of Sciences, S. Kovalevskoi 18, Ekaterinburg \\ 620041, Russia, Département de Chimie Physique, Université de Genève, 30 Quai Ernest Ansermet, \\ 1211 Genève, Switzerland, and Swiss-Norwegian Beamlines at ESRF, 6 rue Horowitz, \\ BP-220, 38043, Grenoble, France
}

Received: April 26, 2010; Revised Manuscript Received: June 15, 2010

\begin{abstract}
To study the reorientational motion of $\mathrm{BH}_{4}$ groups in the low-temperature $(\alpha)$ phase of $\operatorname{Mg}\left(\mathrm{BH}_{4}\right)_{2}$, we have performed nuclear magnetic resonance (NMR) measurements of the ${ }^{1} \mathrm{H}$ and ${ }^{11} \mathrm{~B}$ spin-lattice relaxation rates in this compound over wide ranges of temperature $(82-443 \mathrm{~K})$ and resonance frequency $(14-90 \mathrm{MHz}$ for ${ }^{1} \mathrm{H}$ and $14-28 \mathrm{MHz}$ for $\left.{ }^{11} \mathrm{~B}\right)$. It is found that the thermally activated reorientational motion in $\alpha-\mathrm{Mg}\left(\mathrm{BH}_{4}\right)_{2}$ is characterized by a coexistence of at least three jump processes with strongly differing activation energies. Taking into account the anisotropy of the local environment of $\mathrm{BH}_{4}$ groups in $\alpha-\mathrm{Mg}\left(\mathrm{BH}_{4}\right)_{2}$, these jump processes can be attributed to different types of reorientation. The nearly linear coordination of $\mathrm{BH}_{4}$ groups by two $\mathrm{Mg}$ atoms suggests that the fastest jump process corresponds to the rotation around the 2-fold axis connecting B and two $\mathrm{Mg}$ atoms, whereas the slowest process is associated with the rotation around two other 2-fold axes perpendicular to the $\mathrm{Mg}-\mathrm{B}-\mathrm{Mg}$ line.
\end{abstract}

\section{Introduction}

Magnesium borohydride $\mathrm{Mg}\left(\mathrm{BH}_{4}\right)_{2}$ containing 14.9 mass \% of hydrogen is considered as one of the most promising materials for hydrogen storage. ${ }^{1-5}$ The stability of $\mathrm{Mg}\left(\mathrm{BH}_{4}\right)_{2}$ with respect to thermal decomposition appears to be lower than for other lightweight borohydrides. In fact, pure $\mathrm{Mg}\left(\mathrm{BH}_{4}\right)_{2}$ starts to decompose at around $270{ }^{\circ} \mathrm{C}$, that is, at a much lower temperature than $\mathrm{Li}, \mathrm{Na}$, and $\mathrm{Ca}$ borohydrides. ${ }^{1,6}$ Another advantage of $\mathrm{Mg}\left(\mathrm{BH}_{4}\right)_{2}$ is that the fully dehydrogenated product is a single phase material, $\mathrm{MgB}_{2}{ }^{7,8}$ It has been shown recently that the dehydrogenation-hydrogenation reaction in $\mathrm{Mg}\left(\mathrm{BH}_{4}\right)_{2}$ is partially reversible ${ }^{4,5}$ and that the addition of $5 \mathrm{~mol} \%$ of $\mathrm{TiF}_{3}$ and $\mathrm{ScCl}_{3}$ to $\mathrm{Mg}\left(\mathrm{BH}_{4}\right)_{2}$ significantly accelerates hydrogen desorption. ${ }^{5}$ Elucidation of the crystal structure and hydrogen dynamics in $\mathrm{Mg}\left(\mathrm{BH}_{4}\right)_{2}$ may give a key to improving its hydrogen-storage properties. Recent $\mathrm{X}$-ray ${ }^{9-11}$ and neutron diffraction ${ }^{9}$ studies of the crystal structures of $\mathrm{Mg}\left(\mathrm{BH}_{4}\right)_{2}$ have revealed an unexpected structural complexity of this material. The low-temperature $(\alpha)$ phase of $\mathrm{Mg}\left(\mathrm{BH}_{4}\right)_{2}$ has the hexagonal symmetry. The originally reported ${ }^{9,10}$ structure of this phase (space group $P 6_{1}$ ) was later revised in the space group $P 6_{1} 22$, on the basis of single-crystal diffraction data. ${ }^{11}$ The unit cell of $\alpha-\mathrm{Mg}\left(\mathrm{BH}_{4}\right)_{2}$ contains 330 atoms. Above $463 \mathrm{~K}, \alpha-\mathrm{Mg}\left(\mathrm{BH}_{4}\right)_{2}$ irreversibly transforms to the orthorhombic high-temperature $(\beta)$ phase (space group $F d d d) .{ }^{10,11}$ The unit cell of $\beta-\mathrm{Mg}\left(\mathrm{BH}_{4}\right)_{2}$ contains 704 atoms.

Microscopic information on hydrogen dynamics can be obtained from nuclear magnetic resonance (NMR) and quasielastic neutron scattering (QENS) measurements. Previous NMR ${ }^{12-21}$ and QENS ${ }^{22,23}$ studies of alkali-metal borohydrides $\left(\mathrm{LiBH}_{4}\right.$, $\mathrm{NaBH}_{4}$, and $\mathrm{KBH}_{4}$ ) have revealed two basic types of atomic

* To whom correspondence should be addressed. E-mail: skripov@ imp.uran.ru. Fax: +7-343-374-5244.

${ }^{\dagger}$ Ural Division of the Russian Academy of Sciences.

* Université de Genève.

$\S$ Swiss-Norwegian Beamlines at ESRF. jump motion: the reorientational motion of $\mathrm{BH}_{4}$ groups ${ }^{12-15,17,21-23}$ and the translational diffusion of cations ${ }^{16,20}$ and anions. ${ }^{18,20}$ NMR measurements of spin relaxation rates appear to be especially effective for studies of the reorientational motion in alkali-metal borohydrides, allowing one to trace the $\mathrm{H}$ jump rates over the range of 8 orders of magnitude $\left(\sim 10^{4}\right.$ to $10^{12}$ $\left.\mathrm{s}^{-1}\right) .{ }^{21}$ Hydrogen dynamics in $\mathrm{Mg}\left(\mathrm{BH}_{4}\right)_{2}$ has not been investigated so far. The aim of the present work is to study the $\mathrm{H}$ jump motion in $\alpha-\mathrm{Mg}\left(\mathrm{BH}_{4}\right)_{2}$ using ${ }^{1} \mathrm{H}$ and ${ }^{11} \mathrm{~B}$ NMR measurements of the spectra and spin-lattice relaxation rates over wide ranges of temperature $(82-443 \mathrm{~K})$ and resonance frequency (14-90 MHz). The parameters of $\mathrm{H}$ jump motion derived from our NMR data for $\alpha-\mathrm{Mg}\left(\mathrm{BH}_{4}\right)_{2}$ are discussed in connection with the structural features of this compound.

\section{Experimental Methods}

$\mathrm{Mg}\left(\mathrm{BH}_{4}\right)_{2}$ was prepared using a slightly modified procedure reported by Chłopek et al. ${ }^{3}$ A $60 \mathrm{~mL}$ portion of triethylamineborane was added under a dry nitrogen atmosphere to $5 \mathrm{~g}$ of ball-milled $\mathrm{MgH}_{2}$ (milling time $=2 \mathrm{~h}$ ). The mixture was heated for $1 \mathrm{~h}$ to $100^{\circ} \mathrm{C}$, cooled down and stirred overnight, and heated again for $6 \mathrm{~h}$ to $145^{\circ} \mathrm{C}$. After cooling, $180 \mathrm{~mL}$ of cyclohexane was added, and the mixture was stirred for 2 days. The solid was filtered and dried overnight under vacuum at room temperature, yielding a light gray (almost white) powder. The solvated triethylamine (as seen in IR spectra of the product) was removed by progressive heating over several days under vacuum up to $170{ }^{\circ} \mathrm{C}$, yielding $6.85 \mathrm{~g}$ of $>95 \%$ pure $\mathrm{Mg}\left(\mathrm{BH}_{4}\right)_{2}$. $\mathrm{X}$-ray powder diffraction has confirmed that the dominant phase is $\alpha-\mathrm{Mg}\left(\mathrm{BH}_{4}\right)_{2}$ and revealed small amounts of $\mathrm{Mg}$ and $\mathrm{MgH}_{2}$ ( $\sim 2$ wt \% each). For NMR experiments, the powdered sample was sealed in a glass tube under nitrogen gas.

NMR measurements were performed on a modernized Bruker SXP pulse spectrometer with quadrature phase detection at the frequencies $\omega / 2 \pi=14,23.8$, and $90 \mathrm{MHz}\left(\right.$ for ${ }^{1} \mathrm{H}$ ) and 14 and $28 \mathrm{MHz}\left(\right.$ for ${ }^{11} \mathrm{~B}$ ). The magnetic field was provided by a $2.1 \mathrm{~T}$ 


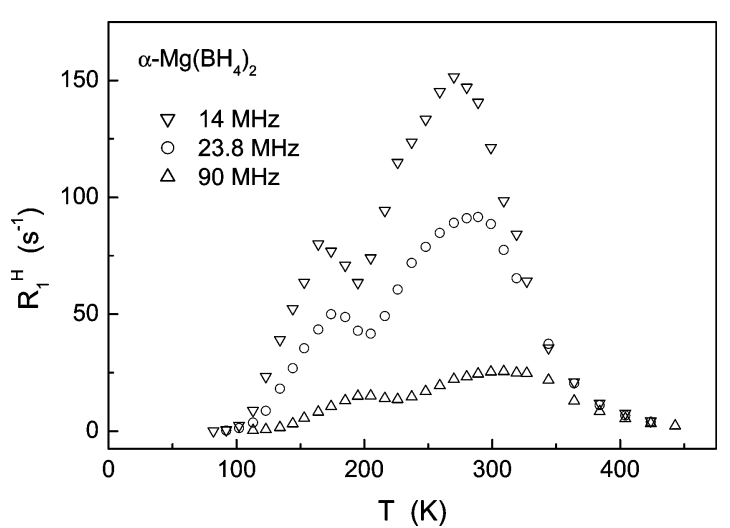

Figure 1. Temperature dependences of the proton spin-lattice relaxation rates measured at $14,23.8$, and $90 \mathrm{MHz}$ for $\alpha-\mathrm{Mg}\left(\mathrm{BH}_{4}\right)_{2}$.

iron-core Bruker magnet. A home-built multinuclear continuouswave NMR magnetometer working in the range of $0.32-2.15$ $\mathrm{T}$ was used for field stabilization. For rf pulse generation, we used a home-built computer-controlled pulse programmer, the PTS frequency synthesizer (Programmed Test Sources, Inc.), and a $1 \mathrm{~kW}$ Kalmus wideband pulse amplifier. Typical values of the $\pi / 2$ pulse length were $2-3 \mu$ s for both ${ }^{1} \mathrm{H}$ and ${ }^{11} \mathrm{~B}$. A probehead with the sample was placed into an Oxford Instruments CF1200 continuous-flow cryostat using nitrogen as a cooling agent. The sample temperature, monitored by a chromel- $(\mathrm{Au}-\mathrm{Fe})$ thermocouple, was stable to $\pm 0.1 \mathrm{~K}$. The nuclear spin-lattice relaxation rates were measured using the saturation-recovery method. NMR spectra were recorded by Fourier transforming the spin echo signals.

\section{Results and Discussion}

The temperature dependences of the proton spin-lattice relaxation rates $R_{1}^{\mathrm{H}}$ measured at three resonance frequencies for $\alpha-\mathrm{Mg}\left(\mathrm{BH}_{4}\right)_{2}$ are shown in Figure 1. In all cases, the recovery of nuclear magnetization is satisfactorily described by a single exponential function. For the relaxation mechanism due to nuclear dipole-dipole interaction modulated by atomic motion, ${ }^{24}$ $R_{1}^{\mathrm{H}}(T)$ typically exhibits a frequency-dependent maximum. This maximum is expected to occur at the temperature at which the atomic jump rate $\tau^{-1}$ becomes nearly equal to the resonance frequency $\omega$. As can be seen from Figure 1, the observed temperature dependence of $R_{1}^{\mathrm{H}}$ for $\alpha-\mathrm{Mg}\left(\mathrm{BH}_{4}\right)_{2}$ shows two wellseparated peaks; both the positions and the amplitudes of these peaks depend on the resonance frequency. Such a behavior suggests a coexistence of at least two atomic jump processes with different characteristic rates $\tau_{i}^{-1}$. Each of the peaks should correspond to the condition $\omega \tau_{i} \approx 1$. The faster jump process gives rise to the low-temperature peak, whereas the slower jump process is responsible for the high-temperature peak. Both jump processes correspond to localized atomic motion and can be attributed to reorientations of the $\mathrm{BH}_{4}$ groups. This is supported by the behavior of the width of the ${ }^{1} \mathrm{H}$ NMR spectra shown in Figure 2 . The line narrowing occurs at considerably lower temperatures $(82-125 \mathrm{~K})$ than the $R_{1}^{\mathrm{H}}$ maxima, as expected. At $T>170 \mathrm{~K}$, the proton line width stops to decrease, being close to $26 \mathrm{kHz}$ over the temperature range of $170-400 \mathrm{~K}$. This rather high plateau value indicates that the motion responsible for the line narrowing is indeed localized because such a motion leads to only partial averaging of the dipole-dipole interactions. For the case of long-range atomic diffusion, one would expect the line narrowing to very small values of the line width. It should be noted that the drop in the ${ }^{1} \mathrm{H}$ NMR line width shown in Figure

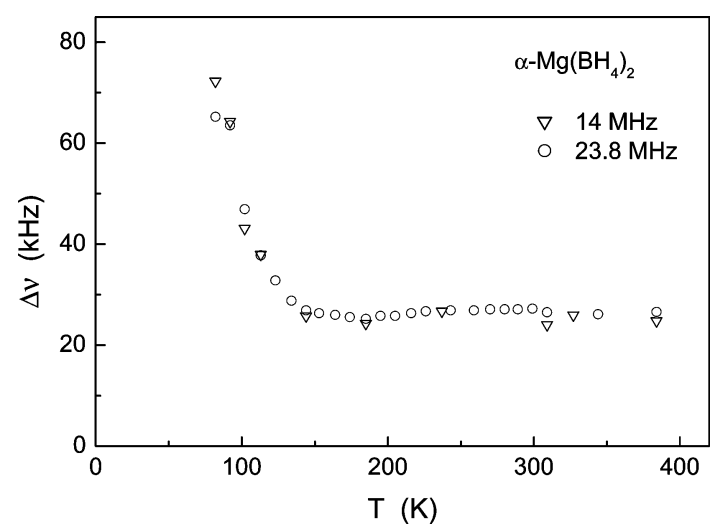

Figure 2. Temperature dependence of the width (full width at halfmaximum) of the proton NMR spectra measured at 14 and $23.8 \mathrm{MHz}$ for $\alpha-\mathrm{Mg}\left(\mathrm{BH}_{4}\right)_{2}$.

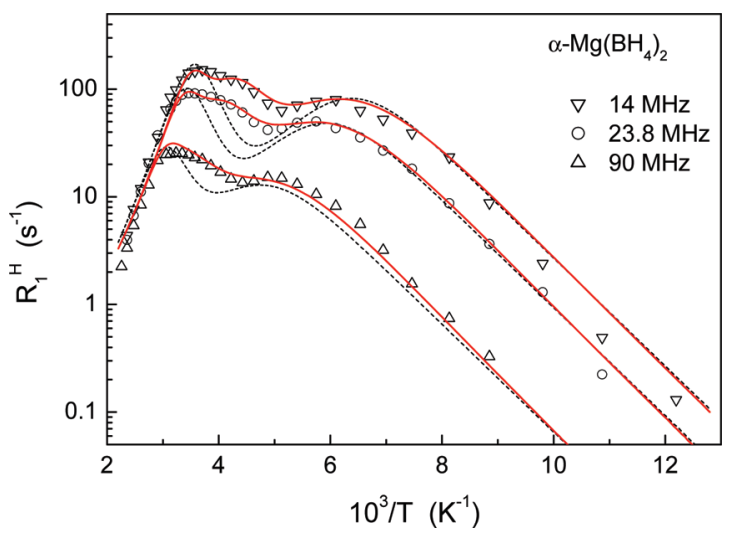

Figure 3. Proton spin-lattice relaxation rates measured at 14, 23.8, and $90 \mathrm{MHz}$ for $\alpha-\mathrm{Mg}\left(\mathrm{BH}_{4}\right)_{2}$ as a function of the inverse temperature. The dashed lines show the simultaneous fits of the "two-peak" model to the data. The red solid lines show the simultaneous fits of the "threepeak" model to the data.

2 is not as sharp as for the previously studied alkali-metal borohydrides. Furthermore, the shape of the ${ }^{1} \mathrm{H}$ NMR spectra at $T<170 \mathrm{~K}$ suggests a presence of several components with different widths. Both of these features are consistent with a coexistence of several jump processes with different rates. At $T>170 \mathrm{~K}$, the rates of all the jump processes are high enough to average out the corresponding parts of the dipole-dipole interaction.

According to the standard theory ${ }^{24}$ of nuclear spin-lattice relaxation due to atomic motion with a single jump rate $\tau^{-1}$, in the limit of slow motion $(\omega \tau \gg 1), R_{1}^{\mathrm{H}}$ should be proportional to $\omega^{-2} \tau^{-1}$, and in the limit of fast motion $(\omega \tau \ll 1), R_{1}^{\mathrm{H}}$ should be proportional to $\tau$ being frequency-independent. If the temperature dependence of $\tau^{-1}$ is governed by the Arrhenius law with the activation energy $E_{\mathrm{a}}$, a plot of $\ln R_{1}^{\mathrm{H}}$ versus $T^{-1}$ should be linear in the limits of both slow and fast motion with the slopes of $-E_{\mathrm{a}} / k_{\mathrm{B}}$ and $E_{\mathrm{a}} / k_{\mathrm{B}}$, respectively. Figure 3 shows the measured proton spin-lattice relaxation rates (at the logarithmic scale) as a function of the inverse temperature. The high-temperature slope of this plot appears to be much steeper than the low-temperature slope. This suggests that the fast and slow reorientational processes in $\alpha-\mathrm{Mg}\left(\mathrm{BH}_{4}\right)_{2}$ are characterized by strongly differing activation energies. The value of $E_{\mathrm{a}}$ estimated from the low-temperature slope in Figure 3 is 100 $\mathrm{meV}$; this value corresponds to the faster jump process. The value of $E_{\mathrm{a}}$ derived from the high-temperature slope in Figure 3 is $310 \mathrm{meV}$ (slower jump process). In Table 1, these values are compared to the activation energies for $\mathrm{BH}_{4}$ reorientations 
TABLE 1: Activation Energies for $\mathrm{BH}_{4}$ Reorientations in Lightweight Borohydrides, As Derived from NMR and QENS Experiments ${ }^{c}$

\begin{tabular}{cllc}
\hline \multicolumn{1}{c}{ compound } & \multicolumn{1}{c}{$\begin{array}{c}\text { activation energy } \\
(\mathrm{meV})\end{array}$} & method & ref \\
\hline $\mathrm{LiBH}_{4}$ (LT phase) & $167(10)$ and $208(10)$ & NMR & 12 \\
& $211(4)$ & NMR & 14 \\
& $182(3)$ and $251(4)$ & NMR & 17 \\
$\mathrm{NaBH}_{4}$ (LT phase) & $154(7)$ & NMR & 12 \\
& $159(4)$ & NMR & 15 \\
& $151(2)$ & NMR & 21 \\
$\mathrm{NaBH}_{4}$ (HT phase) & $117(5)$ & NMR & 12 \\
& $154(4)$ & NMR & 15 \\
& $126(3)$ & NMR & 21 \\
& $117(1)$ & QENS & 22,23 \\
$\mathrm{KBH}_{4}($ HT phase) & $154(4)$ & NMR & 12 \\
& $161(2)$ & NMR & 21 \\
$\left.\mathrm{Mg} \mathrm{BH}_{4}\right)_{2}$ (LT phase) & $100(5)$ and 310 (5) & NMR & this work \\
& $116(6), 198(12)$, and & NMR & this work \\
& $362(5)$ & &
\end{tabular}

$a$ "Two-peak" model. ${ }^{b}$ "Three-peak" model. ${ }^{c}$ Uncertainties in the last digit of $E_{\mathrm{a}}$ are given in parentheses.

obtained from NMR and QENS measurements in alkali-metal borohydrides. It can be seen from Table 1 that all of the values of $E_{\mathrm{a}}$ for alkali-metal borohydrides lie between the two $E_{\mathrm{a}}$ values for $\alpha-\mathrm{Mg}\left(\mathrm{BH}_{4}\right)_{2}$, as derived from the slopes in Figure 3. Before discussing the origin of at least two different energy barriers for $\mathrm{BH}_{4}$ reorientations in $\alpha-\mathrm{Mg}\left(\mathrm{BH}_{4}\right)_{2}$, we shall try to parametrize the $R_{1}^{\mathrm{H}}$ data in terms of the standard theory ${ }^{24}$ of nuclear spin-lattice relaxation due to atomic motion. Our estimates show that the contribution of the ${ }^{1} \mathrm{H}-{ }^{25} \mathrm{Mg}$ dipole-dipole interactions to the measured proton relaxation rates is negligible, and $R_{1}^{\mathrm{H}}$ is dominated by the ${ }^{1} \mathrm{H}-{ }^{11} \mathrm{~B}$ and ${ }^{1} \mathrm{H}-{ }^{1} \mathrm{H}$ interactions. Thus, for the motion characterized by the jump rate $\tau_{i}^{-1}$, the proton spin-lattice relaxation rate can be written as

$R_{1 i}^{\mathrm{H}}=\frac{\Delta M_{\mathrm{HB} i} \tau_{i}}{2}\left[\frac{1}{1+\left(\omega_{\mathrm{H}}-\omega_{\mathrm{B}}\right)^{2} \tau_{i}^{2}}+\frac{3}{1+\omega_{\mathrm{H}}^{2} \tau_{i}^{2}}+\right.$
$\left.\frac{6}{1+\left(\omega_{\mathrm{H}}+\omega_{\mathrm{B}}\right)^{2} \tau_{i}^{2}}\right]+\frac{4 \Delta M_{\mathrm{HH} i} \tau_{i}}{3}\left[\frac{1}{4+\omega_{\mathrm{H}}^{2} \tau_{i}^{2}}+\frac{1}{1+\omega_{\mathrm{H}}^{2} \tau_{i}^{2}}\right]$

where $\omega_{\mathrm{H}}$ and $\omega_{\mathrm{B}}$ are the resonance frequencies of ${ }^{1} \mathrm{H}$ and ${ }^{11} \mathrm{~B}$, respectively, and $\Delta M_{\mathrm{HB} i}$ and $\Delta M_{\mathrm{HH} i}$ are the parts of the dipolar second moment due to $\mathrm{H}-\mathrm{B}$ and $\mathrm{H}-\mathrm{H}$ interactions that are caused to fluctuate by the $i$ th type of motion. We assume that, for each type of the motion, the temperature dependence of $\tau_{i}$ is governed by the Arrhenius law

$$
\tau_{i}=\tau_{0 i} \exp \left(E_{\mathrm{a} i} / k_{\mathrm{B}} T\right)
$$

and

$$
R_{1}^{\mathrm{H}}=\sum_{i} R_{1 i}^{\mathrm{H}}
$$

The parameters of the model are $\Delta M_{\mathrm{HB} i}, \Delta M_{\mathrm{HH} i}, \tau_{0 i}$, and $E_{\mathrm{a} i}$. These parameters can be varied to find the best fit to the $R_{1}^{\mathrm{H}}(T)$ data at the three resonance frequencies simultaneously. Because the $\mathrm{H}-\mathrm{B}$ and $\mathrm{H}-\mathrm{H}$ terms in eq 1 show nearly the same temperature and frequency dependences, it is practically impos- sible to determine the amplitude parameters $\Delta M_{\mathrm{HB} i}$ and $\Delta M_{\mathrm{HH} i}$ independently from the fits. The estimates for alkali-metal borohydrides $^{17,21}$ indicate that $\Delta M_{\mathrm{HB} i}$ and $\Delta M_{\mathrm{HH} i}$ are close to each other. Therefore, for parametrization of the $R_{1}^{\mathrm{H}}$ data, we shall assume that $\Delta M_{\mathrm{HB} i}=\Delta M_{\mathrm{HH} i} \equiv \Delta M_{i}$.

As the first step of an analysis, we can use the two values of the activation energies derived above from the slopes of two peaks. The description of the data in terms of such a "twopeak" model with the fixed values of $E_{\mathrm{a} 1}$ and $E_{\mathrm{a} 2}$ is shown by dashed lines in Figure 3. The corresponding values of the parameters are $\Delta M_{1}=1.3 \times 10^{9} \mathrm{~s}^{-2}, \tau_{01}=7.1 \times 10^{-12} \mathrm{~s}$, and $E_{\mathrm{a} 1}=100 \mathrm{meV}$ (fixed) and $\Delta M_{2}=2.6 \times 10^{9} \mathrm{~s}^{-2}, \tau_{02}=2.9 \times$ $10^{-14} \mathrm{~s}$, and $E_{\mathrm{a} 2}=310 \mathrm{meV}$ (fixed). We assume that the subscript $i=1$ corresponds to the faster type of motion, that is, the one giving rise to the $R_{1}^{\mathrm{H}}$ peak at lower $T$. As can be seen from Figure 3, the "two-peak" model captures the main features of the data. However, the $R_{1}^{\mathrm{H}}$ drop between the peaks predicted by this model appears to be too strong. This suggests the presence of an additional jump process giving rise to an extra relaxation rate peak at intermediate temperatures. It should also be noted that the experimental frequency dependence of $R_{1}^{\mathrm{H}}$ at the low-temperature slope (Figure 3 ) is somewhat weaker than the model frequency dependence. Although this effect may look small, it appears to be systematic. We have verified that, if only the data derived at 14 and $23.8 \mathrm{MHz}$ are used in the fit, the appropriate model curve for $90 \mathrm{MHz}$ lies below the corresponding experimental data over the entire range of the low-temperature slope. Note that there was no such effect for the previously studied alkali-metal borohydrides. ${ }^{17,21}$ The fact that the observed frequency dependence of $R_{1}^{\mathrm{H}}$ is weaker than the expected $\omega^{-2}$ law often indicates the presence of a certain distribution of jump rates (or activation energies) ${ }^{25}$ for the corresponding jump process.

Therefore, as the next step of parametrization, we use a "threepeak" model, including three types of jump motion and a Gaussian distribution of activation energies for the fastest jump process. The parameters of this model are $\Delta M_{i}, \tau_{0 i}, E_{\mathrm{a} i}(i=1$, 2,3 ), and the width (dispersion) of the activation energy distribution $\Delta E_{\mathrm{a} 1}$. Again, we look for a set of parameters giving the best fit to the $R_{1}^{\mathrm{H}}(T)$ data at the three resonance frequencies simultaneously. The results of this fit are shown by red solid lines in Figure 3. The values of the amplitude parameters resulting from the fit are $\Delta M_{1}=1.5 \times 10^{9} \mathrm{~s}^{-2}, \Delta M_{2}=1.6 \times$ $10^{9} \mathrm{~s}^{-2}$, and $\Delta M_{3}=1.7 \times 10^{9} \mathrm{~s}^{-2}$, and the corresponding motional parameters are $\tau_{01}=(2.3 \pm 0.1) \times 10^{-12} \mathrm{~s}, E_{\mathrm{a} 1}=$ $116 \pm 6 \mathrm{meV}$, and $\Delta E_{\mathrm{a} 1}=10 \pm 4 \mathrm{meV} ; \tau_{02}=(5.7 \pm 0.5) \times$ $10^{-13} \mathrm{~s}$ and $E_{\mathrm{a} 2}=198 \pm 12 \mathrm{meV}$; and $\tau_{03}=(4.0 \pm 0.1) \times$ $10^{-15} \mathrm{~s}$ and $E_{\mathrm{a} 3}=362 \pm 5 \mathrm{meV}$. The uncertainties of the fit parameters correspond to one standard deviation, as given by the nonlinear fitting procedure. The high accuracy of the motional parameters for the fastest and the slowest jump processes originates from wide dynamic ranges of the data at the low- $T$ and high- $T$ slopes, respectively. For the intermediate jump process, the accuracy of the fit parameters is poorer. The values of $E_{\mathrm{a} i}$ for the "three-peak" model are also included in Table 1. It should be noted that the resulting value of the average activation energy for the fastest jump process, $E_{\mathrm{a} 1}$, appears to be somewhat higher than that estimated from the low-temperature slope of the $R_{1}^{\mathrm{H}}(T)$ data. This feature originates from the activation energy distribution used in our model. In fact, it is known $^{25}$ that such a distribution makes the low-temperature slope of $R_{1}^{\mathrm{H}}(T)$ less steep than that described by the average activation energy. The resulting value of the activation energy for the slowest jump process, $E_{\mathrm{a} 3}$, is also higher than that 


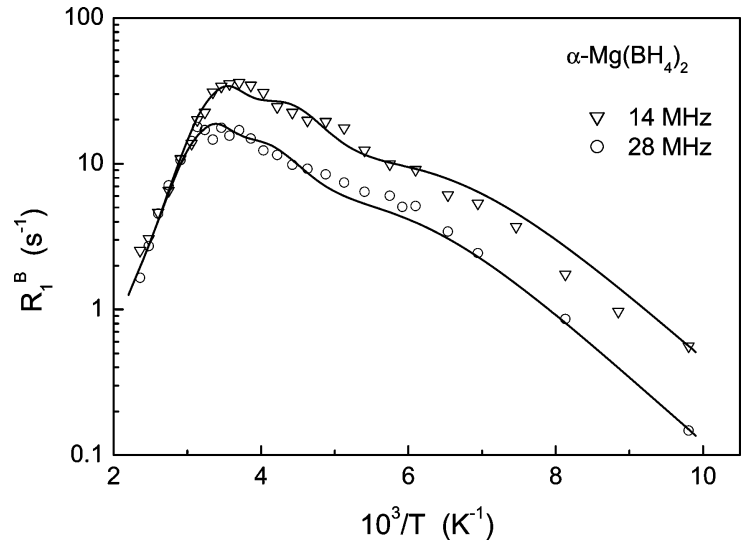

Figure 4. ${ }^{11} \mathrm{~B}$ spin-lattice relaxation rates measured at 14 and $28 \mathrm{MHz}$ for $\alpha-\operatorname{Mg}\left(\mathrm{BH}_{4}\right)_{2}$ as a function of the inverse temperature. The solid lines show the simultaneous fits of the "three-peak" model to the data.

estimated from the high-temperature slope of the $R_{1}^{\mathrm{H}}(T)$ data. This is related to counter-balancing the effect of the intermediate jump process $(i=2)$ on the high-temperature slope of $R_{1}^{\mathrm{H}}(T)$. The difference in jump rates of different reorientation processes appears to be quite large, especially at low temperatures. For example, at $T=170 \mathrm{~K}$, estimates based on the parameters of the "three-peak" model yield $\tau_{1}^{-1}=1.5 \times 10^{8} \mathrm{~s}^{-1}$ (the most probable value), $\tau_{2}^{-1}=2.3 \times 10^{6} \mathrm{~s}^{-1}$, and $\tau_{3}^{-1}=4.7 \times 10^{4} \mathrm{~s}^{-1}$. Although the dispersion $\Delta E_{\mathrm{a} 1}$ of the activation energy distribution for the fastest jump process is only $\sim 9 \%$ of the average value of $E_{\text {al }}$, the width of the corresponding jump rate distribution is not small at all, especially at low temperatures. At $170 \mathrm{~K}$, the values of $\tau_{1}^{-1}$ vary within the $\pm 2 \sigma$ interval from $3.8 \times 10^{7}$ to $6.3 \times 10^{8} \mathrm{~s}^{-1}$.

The temperature dependences of the ${ }^{11} \mathrm{~B}$ spin-lattice relaxation rates $R_{1}^{\mathrm{B}}$ measured at two resonance frequencies are shown in Figure 4. Comparison of Figures 3 and 4 indicates that general features of the behavior of the ${ }^{11} \mathrm{~B}$ relaxation rates in $\alpha-\mathrm{Mg}$ $\left(\mathrm{BH}_{4}\right)_{2}$ are similar to those of the proton relaxation rates, although the overall quality of the ${ }^{11} \mathrm{~B}$ data is somewhat poorer. As in the case of alkali-metal borohydrides, ${ }^{13,17}$ the quadrupole contribution to the ${ }^{11} \mathrm{~B}$ relaxation rate in $\mathrm{Mg}\left(\mathrm{BH}_{4}\right)_{2}$ should be small, and the dominant contribution to this rate originates from the $\mathrm{B}-\mathrm{H}$ dipole-dipole interaction modulated by reorientations of $\mathrm{BH}_{4}$ tetrahedra. This dominant contribution for each jump process can be written as

$$
\begin{array}{r}
R_{1 i}^{\mathrm{B}}=\frac{\Delta M_{\mathrm{BH} i} \tau_{i}}{2}\left[\frac{1}{1+\left(\omega_{\mathrm{B}}-\omega_{\mathrm{H}}\right)^{2} \tau_{i}^{2}}+\frac{3}{1+\omega_{\mathrm{B}}^{2} \tau_{i}^{2}}+\right. \\
\left.\frac{6}{1+\left(\omega_{\mathrm{B}}+\omega_{\mathrm{H}}\right)^{2} \tau_{i}^{2}}\right]
\end{array}
$$

where $\Delta M_{\mathrm{BH} i}$ is the part of the ${ }^{11} \mathrm{~B}$ dipolar second moment due to $\mathrm{B}-\mathrm{H}$ interaction that is caused to fluctuate by the $i$ th jump process. For parametrization of the ${ }^{11} \mathrm{~B}$ relaxation data, it is natural to use the same "three-peak" model as for the proton relaxation. This model is based on eqs 4 and 2 and the boron replica of eq 3 with $i=1,2,3$ and a Gaussian distribution of the activation energies for the fastest jump process. To reduce the number of fit parameters, we assume that the values of $E_{\mathrm{a} i}$ are the same as those derived from the analysis of the ${ }^{1} \mathrm{H}$ relaxation data. The results of the simultaneous fit of the "threepeak" model to the $R_{1}^{\mathrm{B}}(T)$ data at two resonance frequencies

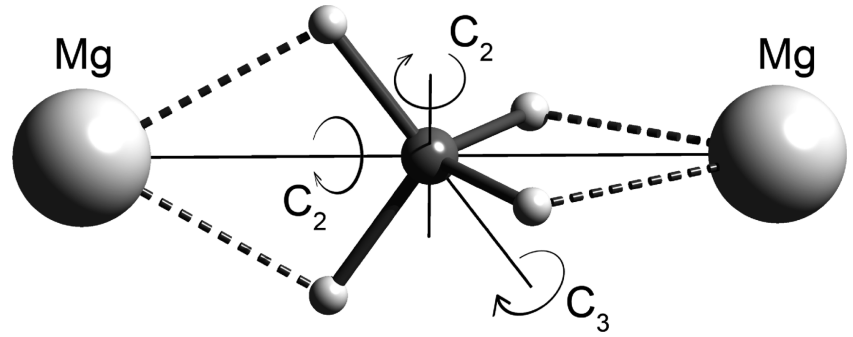

Figure 5. Schematic view of the coordination environment of $\mathrm{BH}_{4}$ groups in $\alpha-\mathrm{Mg}\left(\mathrm{BH}_{4}\right)_{2}$ : large spheres, $\mathrm{Mg}$ atoms; medium spheres, $\mathrm{B}$; and small spheres, $\mathrm{H}$. The $\mathrm{Mg}-\mathrm{H}$ bonds are shown by the bold dashed lines. $C_{2}$ and $C_{3}$ are the 2-fold and 3-fold reorientation axes of the $\mathrm{BH}_{4}$ group, respectively.

are shown by solid lines in Figure 4. The resulting amplitude parameters are $\Delta M_{\mathrm{BH} 1}=9.0 \times 10^{8} \mathrm{~s}^{-2}, \Delta M_{\mathrm{BH} 2}=1.8 \times 10^{9}$ $\mathrm{s}^{-2}$, and $\Delta M_{\mathrm{BH} 3}=1.8 \times 10^{9} \mathrm{~s}^{-2}$, and the corresponding motional parameters are $\tau_{01}=(2.3 \pm 0.2) \times 10^{-12} \mathrm{~s}, E_{\mathrm{a} 1}=116 \mathrm{meV}$ (fixed), and $\Delta E_{\mathrm{a} 1}=15 \pm 6 \mathrm{meV} ; \tau_{02}=(4.7 \pm 0.5) \times 10^{-13} \mathrm{~s}$ and $E_{\mathrm{a} 2}=198 \mathrm{meV}$ (fixed); and $\tau_{03}=(4.0 \pm 0.3) \times 10^{-15} \mathrm{~s}$ and $E_{\mathrm{a} 3}=362 \mathrm{meV}$ (fixed). Thus, the ${ }^{11} \mathrm{~B}$ spin-lattice relaxation data for $\alpha-\operatorname{Mg}\left(\mathrm{BH}_{4}\right)_{2}$ can be satisfactorily described by the set of motional parameters that are close to those obtained from the ${ }^{1} \mathrm{H}$ relaxation results.

What is the origin of a coexistence of strongly differing activation energies for $\mathrm{BH}_{4}$ reorientations in $\alpha-\mathrm{Mg}\left(\mathrm{BH}_{4}\right)_{2}$ ? Taking into account the structural complexity of this compound, one may assume that its six crystallographically inequivalent $\mathrm{BH}_{4}$ groups ${ }^{11}$ have different energy barriers for reorientations. However, all $\mathrm{BH}_{4}$ groups have similar local environments ${ }^{11}$ so that it is hardly possible to attribute strongly differing values of $E_{\text {a } i}$ to different $\mathrm{BH}_{4}$ groups. The remarkable feature of the local environments of $\mathrm{BH}_{4}$ groups is their nearly linear coordination by two $\mathrm{Mg}$ atoms, as schematically shown in Figure 5. Such an unusual coordination of $\mathrm{BH}_{4}$ groups immediately suggests that different values of $E_{\mathrm{a} i}$ can be related to different types of reorientation of the same $\mathrm{BH}_{4}$ group. In fact, the rotation around the 2-fold axis connecting $\mathrm{B}$ and $\mathrm{Mg}$ atoms (horizontal $C_{2}$ axis in Figure 5) does not break any $\mathrm{Mg}-\mathrm{H}$ bonds; therefore, this type of motion should correspond to the lowest value of $E_{\mathrm{a}}$. The rotation around two other 2-fold axes of a $\mathrm{BH}_{4}$ tetrahedron (one of which is shown as the vertical $C_{2}$ axis in Figure 5) breaks all the $\mathrm{Mg}-\mathrm{H}$ bonds and should be associated with the highest value of $E_{\mathrm{a}}$. The intermediate value of $E_{\mathrm{a}}$ can be ascribed to the rotation around the 3-fold axes (one of which is shown as $C_{3}$ in Figure 5) because such a rotation breaks half of the $\mathrm{Mg}-\mathrm{H}$ bonds. It should be noted that Figure 5 represents the "idealized" environment of $\mathrm{BH}_{4}$ groups in $\alpha-\mathrm{Mg}\left(\mathrm{BH}_{4}\right)_{2}$. In the real structure, the $\mathrm{Mg}-\mathrm{B}-\mathrm{Mg}$ angles vary in the range of $148-177^{\circ}$ for different $\mathrm{BH}_{4}$ groups. ${ }^{11}$ These variations are expected to lead to the appearance of a certain distribution of the energy barriers, in agreement with our experimental data. Thus, the main features of the coexisting energy barriers in $\alpha-\mathrm{Mg}\left(\mathrm{BH}_{4}\right)_{2}$ can be qualitatively explained in terms of the anisotropic coordination of $\mathrm{BH}_{4}$ groups. These results are consistent with the idea ${ }^{21,23}$ that the energy barriers for $\mathrm{BH}_{4}$ reorientations in borohydrides are determined primarily by metal $-\mathrm{H}$ interactions.

The value of $E_{\mathrm{a}}$ for the slowest jump process, $E_{\mathrm{a} 3}=362 \mathrm{meV}$, appears to be the highest activation energy for reorientational motion in borohydrides studied so far. To compare this value with other independent results, we may use two approaches. The first one is based on the results of recent first-principles calculations ${ }^{26}$ for $\operatorname{Mg}\left(\mathrm{BH}_{4}\right)_{2}$. The most straightforward way to 
compare our result for $E_{\mathrm{a} 3}$ with these calculations is to use the calculated total energy difference for different orientations of $\mathrm{BH}_{4}$ groups with respect to tetrahedrally coordinated $\mathrm{Mg}$ cations. For the actual $\mathrm{Mg}-\mathrm{B}$ distance of $2.40 \AA$ in $\mathrm{Mg}\left(\mathrm{BH}_{4}\right)_{2}$, the calculated total energy difference between the bidentate and tridentate orientations of $\mathrm{BH}_{4}$ groups ${ }^{26}$ is about $0.35 \mathrm{eV}$. This value is close to our value of $E_{\mathrm{a} 3}$. The second approach is to use the known approximate relation ${ }^{27}$ between the height of the reorientational barrier and the libration frequency $v_{\text {lib }}$ of $\mathrm{BH}_{4}$ groups. A rough estimate based on this relation shows that our value of $E_{\mathrm{a} 3}$ should correspond to a $\nu_{\text {lib }}$ of $545 \mathrm{~cm}^{-1}$. A strong peak at $\sim 525 \mathrm{~cm}^{-1}$ due to librations of $\mathrm{BH}_{4}$ groups has been found in inelastic neutron scattering measurements ${ }^{28}$ for $\mathrm{Mg}$ $\left(\mathrm{BH}_{4}\right)_{2}$, in good agreement with the predicted value.

\section{Conclusions}

The analysis of the temperature and frequency dependences of the measured ${ }^{1} \mathrm{H}$ and ${ }^{11} \mathrm{~B}$ spin-lattice relaxation rates for $\alpha-\mathrm{Mg}\left(\mathrm{BH}_{4}\right)_{2}$ has revealed the parameters of reorientational motion of $\mathrm{BH}_{4}$ groups in this compound. It is found that, in contrast to alkali-metal borohydrides, the reorientational motion in $\alpha-\mathrm{Mg}\left(\mathrm{BH}_{4}\right)_{2}$ exhibits a coexistence of at least three jump processes with strongly differing activation energies. The frequency dependence of the ${ }^{1} \mathrm{H}$ and ${ }^{11} \mathrm{~B}$ spin-lattice relaxation rates also suggests that the fastest jump process is characterized by a certain distribution of activation energies. The values of $E_{\mathrm{a}}$ derived from our data are $116 \pm 6 \mathrm{meV}$ (average value for the fastest jump process), $198 \pm 12 \mathrm{meV}$ (for the intermediate process), and $362 \pm 5 \mathrm{meV}$ (for the slowest process). The nearly linear coordination of $\mathrm{BH}_{4}$ groups by two $\mathrm{Mg}$ atoms suggests that the fastest jump process corresponds to the rotation around the 2-fold axis connecting $\mathrm{B}$ and $\mathrm{Mg}$ atoms, whereas the slowest process is associated with the rotations around two other 2-fold axes perpendicular to the $\mathrm{B}-\mathrm{Mg}$ line. The intermediate jump process can be attributed to the rotations around 3-fold axes.

Acknowledgment. This work was partially supported by the Priority Program "Basics of Development of Energy Systems and Technologies" of the Russian Academy of Sciences and by the Swiss National Science Foundation.

\section{References and Notes}

(1) Nakamori, Y.; Miwa, K.; Ninomiya, A.; Li, H.; Ohba, N.; Towata, S.; Züttel, A.; Orimo, S. Phys. Rev. B 2006, 74, 045126.
(2) Orimo, S.; Nakamori, Y.; Eliseo, J. R.; Züttel, A.; Jensen, C. M. Chem. Rev. 2007, 107, 4111.

(3) Chłopek, K.; Frommen, C.; Léon, A.; Zabara, O.; Fichtner, M. J. Mater. Chem. 2007, 17, 3496.

(4) Severa, G.; Rönnebro, E.; Jensen, C. M. Chem. Commun. 2010, $46,421$.

(5) Newhouse, R. J.; Stavila, V.; Hwang, S.-J.; Klebanoff, L. E.; Zhang, J. Z. J. Phys. Chem. C 2010, 114, 5224.

(6) Nakamori, Y.; Li, H. W.; Miwa, K.; Towata, S.; Orimo, S. Mater. Trans. 2006, 47, 1898.

(7) Hanada, N.; Chłopek, K.; Frommen, C.; Lohstroh, W.; Fichtner, M. J. Mater. Chem. 2008, 18, 2611.

(8) Soloveichik, G. L.; Gao, Y.; Rijssenbeek, J.; Andrus, M.; Kniajanski, S.; Bowman, R. C.; Hwang, S.-J.; Zhao, J. C. Int. J. Hydrogen Energy 2009, 34, 916.

(9) Cerný, R.; Filinchuk, Y.; Hagemann, H.; Yvon, K. Angew. Chem., Int. Ed. 2007, 46, 5765.

(10) Her, J.-H.; Stephens, P. W.; Gao, Y.; Soloveichik, G. L.; Rijssenbeek, J.; Andrus, M.; Zhao, J.-C. Acta Crystallogr., B 2007, 63, 561. 925 .

(12) Tsang, T.; Farrar, T. C. J. Chem. Phys. 1969, 50, 3498.

(13) Niemelä, L.; Ylinen, E. Phys. Lett. 1970, 31A, 369.

(14) Tarasov, V. P.; Bakum, S. I.; Privalov, V. I.; Shamov, A. A. Russ. J. Inorg. Chem. 1990, 35, 1035.

(15) Tarasov, V. P.; Bakum, S. I.; Privalov, V. I.; Shamov, A. A. Russ. J. Inorg. Chem. 1990, 35, 2096.

(16) Matsuo, M.; Nakamori, Y.; Orimo, S.; Maekawa, H.; Takamura, H. Appl. Phys. Lett. 2007, 91, 224103.

(17) Skripov, A. V.; Soloninin, A. V.; Filinchuk, Y.; Chernyshov, D. J. Phys. Chem. C 2008, 112, 18701.

(18) Corey, R. L.; Shane, D. T.; Bowman, R. C.; Conradi, M. S. J. Phys. Chem. C 2008, 112, 18706.

(19) Shane, D. T.; Bowman, R. C.; Conradi, M. S. J. Phys. Chem. C 2009, 113, 5039.

(20) Soloninin, A. V.; Skripov, A. V.; Buzlukov, A. L.; Stepanov, A. P. J. Solid State Chem. 2009, 182, 2357.

(21) Babanova, O. A.; Soloninin, A. V.; Stepanov, A. P.; Skripov, A. V.; Filinchuk, Y. J. Phys. Chem. C 2010, 114, 3712.

(22) Remhof, A.; Łodziana, Z.; Buchter, F.; Martelli, P.; Pendolino, F.; Friedrichs, O.; Züttel, A.; Embs, J. P. J. Phys. Chem. C 2009, 113, 16834.

(23) Remhof, A.; Gremaud, R.; Buchter, F.; Łodziana, Z.; Embs, J. P.; Ramirez-Cuesta, T. A. J.; Borgschulte, A.; Züttel, A. Z. Phys. Chem. 2010, 224, 263.

(24) Abragam, A. The Principles of Nuclear Magnetism; Clarendon Press: Oxford, U.K., 1961.

(25) Markert, J. T.; Cotts, E. J.; Cotts, R. M. Phys. Rev. B 1988, 37, 6446.

(26) Łodziana, Z.; Van Setten, M. J. Phys. Rev. B 2010, 81, 024117.

(27) Gutowsky, H. S.; Pake, G. E.; Bersohn, R. J. Chem. Phys. 1954, 22,643 .

(28) Giannasi, A.; Colognesi, D.; Ulivi, L.; Zoppi, M.; Ramirez-Cuesta, A. J.; Bardají, E. G.; Roehm, E.; Fichtner, M. J. Phys. Chem. A 2010, 114, 2788.

JP1037206 